\title{
Moderate Resolution Imaging Spectroradiometer on Terra: limitations for ocean color applications
}

\author{
Bryan A. Franz ${ }^{a, b}$, Ewa J. Kwiatkowska ${ }^{a, b}$, Gerhard Meister ${ }^{a, c}$, \\ and Charles R. McClain ${ }^{\mathrm{a}, \mathrm{d}}$ \\ aNASA Ocean Biology Processing Group, Greenbelt, MD, USA \\ ${ }^{\mathrm{b}}$ Science Applications International Corporation, Beltsville, MD, USA \\ ${ }^{\mathrm{c}}$ Futuretech Corporation, Greenbelt, MD, USA \\ ${ }^{\mathrm{d}}$ National Aeronautics and Space Administration, Greenbelt, MD, USA
}

\begin{abstract}
The Moderate Resolution Imaging Spectroradiometer (MODIS) is currently flying on both the Terra and Aqua satellite platforms. The Ocean Biology Processing Group (OBPG) at NASA Goddard Space Flight Center is producing operational ocean color products from the MODIS-Aqua sensor; however, documented uncertainties and instabilities in the prelaunch and on-orbit characterization have inhibited the production of similar products from MODISTerra. In particular, the radiometric response of the 412-nm band has degraded by more than $40 \%$ over the 7 -year mission lifespan, with similar though less extreme changes in the longer wavelengths. While such variability may be fully correctable through the on-board calibration system, it suggests that the optical properties of the scan mirror have changed significantly since launch. Furthermore, the degradation trends are substantially different between the two mirror sides, which is likely a result of asymmetric damage done to the mirror during prelaunch testing. These effects contribute to uncertainty in our knowledge of instrument response versus incidence angle on the mirror and sensitivity with respect to polarization of the observed radiance. In this paper, we examine the impact of apparent MODIS-Terra instrument characterization errors on the derived ocean color products and show that residual errors in the current operational calibration give rise to significant cross-scan artifacts, mirror-side differences, and detector-to-detector striping in the retrieved water-leaving radiances. In addition, we describe OBPG efforts to reduce these artifacts through statistical and vicarious instrument characterization, and show the quality of the resulting water-leaving radiance retrievals relative to those derived from MODIS-Aqua.
\end{abstract}

Keywords: ocean color, remote sensing, calibration, validation, MODIS, SeaWiFS

\section{INTRODUCTION}

The Moderate Resolution Imaging Spectroradiometer (MODIS) [1] is an Earth-viewing, satellitebased global imager that measures visible and infrared radiance over the spectral range from 0.4 to $14.4 \mu \mathrm{m}$ to support land, atmosphere, and ocean applications. A MODIS sensor has been operating from the Terra platform of NASA's Earth Observing System constellation of satellites since December 1999, with a second MODIS launched on the Aqua platform in May 2002. Of the 36 spectral channels on MODIS, 9 bands in the visible and near infrared (NIR) spectral regime, with center wavelengths from $412 \mathrm{~nm}$ to $869 \mathrm{~nm}$, were specifically designed with the enhanced radiometric sensitivity required for ocean color applications. Such applications seek to measure the spectral distribution of up-welling visible light just above the ocean surface, which is referred to as the water-leaving radiance, $L_{w}(\lambda)$, for sensor wavelengths $\lambda$. This spectral information can be used to derive various geophysical properties of the water, such as concentrations of the phytoplankton pigment chlorophyll $a, C_{a}$.

Satellite-borne ocean color instruments, however, actually measure the total radiance, $L_{t}(\lambda)$, exiting the top of the atmosphere (TOA), for which the dominant signal is the light scattered 
Table 1. Ratios of Observed Radiance to Water-Leaving Radiance by Waters Class.

\begin{tabular}{ccccc}
\hline $\begin{array}{c}\text { Wavelength } \\
\mathrm{nm}\end{array}$ & $\begin{array}{c}\text { Deep Water } \\
\text { depth }>1000 \mathrm{~m}\end{array}$ & $\begin{array}{c}\text { Oligotrophic } \\
C_{a}<0.1 \mathrm{mg} \mathrm{m}^{-3}\end{array}$ & $\begin{array}{c}\text { Mesotrophic } \\
0.1<C_{a}<1\end{array}$ & $\begin{array}{c}\text { Eutrophic } \\
C_{a}>1 \mathrm{mg} \mathrm{m}^{-3}\end{array}$ \\
\hline 412 & 7.3 & 5.7 & 9.3 & 24.0 \\
443 & 7.0 & 5.8 & 8.4 & 15.1 \\
488 & 6.6 & 6.0 & 7.1 & 8.7 \\
531 & 10.7 & 11.6 & 9.8 & 6.5 \\
551 & 13.3 & 15.0 & 11.8 & 6.2 \\
667 & 76.5 & 95.8 & 64.4 & 14.6 \\
678 & 70.5 & 97.2 & 57.7 & 14.0 \\
\hline
\end{tabular}

by air molecules and aerosols. $L_{w}(\lambda)$ is retrieved from observed $L_{t}(\lambda)$ through a process of atmospheric correction (e.g.: Gordon and Wang, 1994) [2]. For a complete discussion of the atmospheric correction algorithm employed by the OBPG, we refer the reader to Franz et al. (2007) [3] and references therein, but for simplicity here we write

$$
t L_{w}(\lambda)=L_{t}(\lambda)-L_{a t m}(\lambda)
$$

where $L_{a t m}(\lambda)$ represents all contributions from the atmosphere and $t$ is a quantity close to unity that represents transmittance of the diffuse water-leaving radiance through the atmosphere. Table 1 provides typical values for the ratios of $L_{t}(\lambda)$ to $L_{w}(\lambda)$ for various water classifications, including deep water (depth $>1000$ meters), very clear oligotrophic waters, mesotrophic waters with moderate $C_{a}$ concentrations, and eutrophic waters generally associated with coastal and inland locales. The radiance ratios were derived by averaging globally distributed ocean observations for each water class, as collected under cloud-free skies for a typical day. The Table is provided here to indicate how small errors in the calibration of the observed radiance, $L_{t}(\lambda)$, are multiplied in the retrieval of $L_{w}(\lambda)$ and derived bio-optical properties. For example, a $1 \%$ error in $L_{t}(551)$ would give rise to a 6-15\% error in $L_{w}(551)$, with the largest fractional error occurring in clear (blue) water, while a $1 \%$ calibration error in $L_{t}(443)$ would produce a 6-15\% error in $L_{w}$ (443), with the largest error in eutrophic (green) water. This variation in calibration sensitivity with wavelength is further compounded in the operational $C_{a}$ algorithms [4] employed for MODIS, which make use of $L_{w}(\lambda)$ to $L_{w}(551)$ ratios.

In the sections that follow, we provide some details of the MODIS instrument characterization and calibration, both prelaunch and on-orbit, and discuss specific issues and limitations that may contribute to error in the retrieval of $L_{w}(\lambda)$ from MODIS-Terra. This is followed by a detailed trend analysis of the MODIS-Terra ocean color retrievals that is designed to reveal temporal and geometry-dependent variabilities that may be indicative of instrument calibration or characterization errors. Our analysis includes comparison with ocean color retrievals from MODIS-Aqua, as well as those from the Sea-viewing Wide Field-of-view Sensor (SeaWiFS) [5]. The data from all three sensors were processed by the Ocean Biology Processing Group (OBPG) using common software and algorithms [6] to minimize differences in the determination of $L_{a t m}(\lambda)$. We conclude by describing a number of approaches currently being developed to improve the performance of MODIS-Terra for ocean color applications.

\section{INSTRUMENT CALIBRATION AND RADIOMETRIC STABILITY}

Primary responsibility for MODIS instrument calibration rests with the MODIS Calibration Support Team (MCST), who developed the calibration procedures and continue to provide routine updates to the calibration coefficients based on the on-board calibration (OBC) system. We refer the reader to Xiong and Barnes (2006) [7] for details on the MCST calibration, but we 
Table 2. MODIS scan geometry. Relationship between scan pixel number, scan angle (SA), mirror incidence angle (AOI), space view (SV), and solar diffuser (SD) view within a physical MODIS scan.

\begin{tabular}{lccccc}
\hline & Start & SV & Nadir & SD & End \\
\hline Scan Pixel & 1 & 23 & 677.5 & 979 & 1354 \\
Scan Angle & -55.0 & -53.2 & 0.0 & 24.5 & 55.00 \\
Mirror AOI & $10.5^{\circ}$ & $11.4^{\circ}$ & $38.0^{\circ}$ & $50.3^{\circ}$ & $65.5^{\circ}$ \\
\hline
\end{tabular}

provide a limited discussion of the prelaunch and on-orbit characterization to highlight some concerns with the use of MODIS-Terra for ocean color applications.

One critical element of the prelaunch characterization is the determination of radiometric response versus scan angle (RVS). As the satellite orbits the Earth, the instrument employs a two-sided rotating scan mirror to collect a continuous swath of the surface (1354 pixels at approximately $1-\mathrm{km} \times 1-\mathrm{km}$ nadir ground resolution). During a scan, the angle of incidence (AOI) of the observed radiance on the mirror increases monotonically (Table 2), and a prelaunch characterization analysis was performed to assess variation in mirror reflectance with this change in AOI. A similar analysis was also performed to evaluate the sensitivity of the instrument response to the polarization of the incoming light. In the context of ocean color processing, it must be considered that light scattered by the atmosphere is polarized, where the state of polarization varies with viewing and solar radiant path geometries [8] (where view path is related to the scan angle and thus AOI). The polarization sensitivity of the MODIS instrument is significant [9] and must be accounted for in the retrieval of $L_{w}(\lambda)$ from $L_{t}(\lambda)$.

Unfortunately, for MODIS-Terra, the optical characteristics of the scan mirror may have been altered subsequent to the instrument RVS characterization effort. During a special sensor characterization activity, the nadir door became too hot and a small amount of paint may have evaporated and deposited on the scan mirror (X. Xiong, personal communication). The mirror was cleaned, but the event raises suspicion that the prelaunch characterization of the MODISTerra mirror may not adequately represent the at-launch configuration.

MCST uses the OBC system on MODIS to assess temporal change in system response relative to the at-launch configuration. For both MODIS sensors, changes in responsivity are tracked on-orbit through periodic observations of the Moon through the space-view (SV) port and the Sun by way of a solar diffuser (SD). The SV is also used to track radiometric offsets through dark-sky observations, but in this discussion we use SV to indicate lunar observations, exclusively. Fig. 1 shows the MCST-derived trends in SV and SD observations over the Terra mission for the 412 and 551-nm bands, with trends for MODIS-Aqua also provided for comparison. The 412-nm band has been found to exhibit the largest change in responsivity among the ocean bands of either MODIS sensor. For MODIS-Terra, this band has degraded by more than $40 \%$ since launch, and the amount of change differs significantly between mirror-side 1 (MS1) and mirror-side 2 (MS2). As of the beginning of 2007, the mirror-side reflectivity difference has grown to more than 5\% (compare to 1\% for Aqua). This asymmetric degradation in reflectivity between the two MODIS-Terra mirror-sides is likely due to the prelaunch over-heating event, which supports the suspicion that the mirror characteristics were modified by that event. Tables 3 and 4 summarize changes in radiometric response of MODIS on Terra and Aqua, respectively, as of January 2007, based on the OBC measurements.

In principle, the temporal changes in instrument response can be corrected by application of the SD trends to the Earth-view observations, but it is important to recognize that the required corrections are very large (Table 3 ), and any residual errors in $L_{t}(\lambda)$ will be multiplied in $L_{w}(\lambda)$ by a factor of 5 or more (Table 1). Furthermore, the trends in the SD and SV measurements, as interpreted by MCST, indicate that the degradation is not continuous and well 

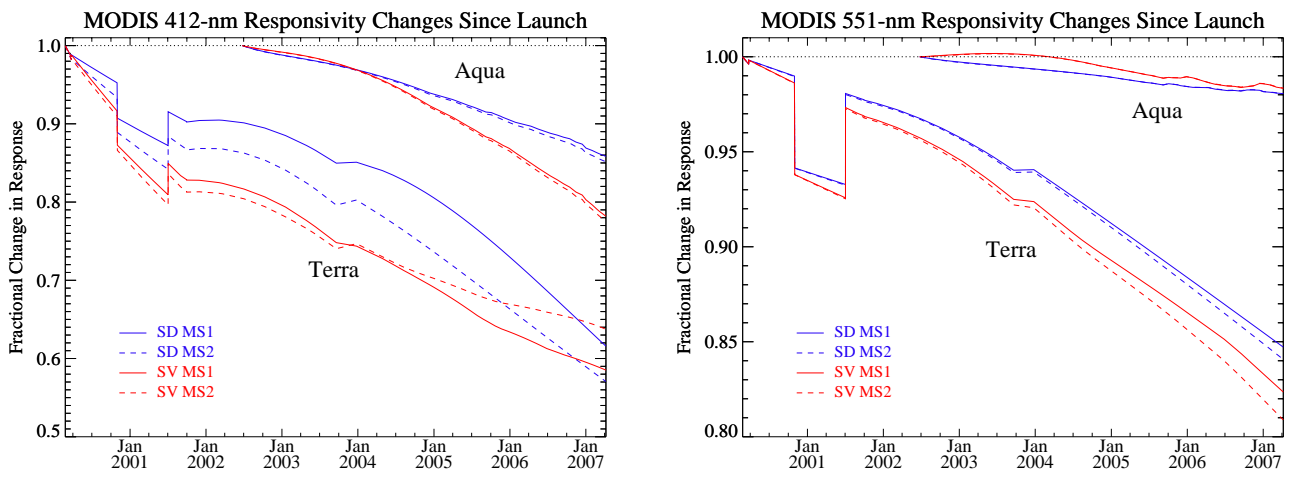

Fig. 1. MODIS-Terra and MODIS-Aqua sensor response changes since first on-orbit calibration. Trends are for ocean bands centered at 412 and $551 \mathrm{~nm}$, as derived by MCST from observations of the Sun via the solar diffuser (SD) and the Moon via the space view (SV) port. Results for mirror-side 1 (MS1) are shown as solid lines, with mirror-side 2 (MS2) as dashed lines. (Terra calibration is version 5.0.6.27OC, and Aqua calibration is version 5.0.7.22. SD trends have been corrected for degradation of the diffuser plate).

behaved. Some aspects of this variability are well understood, such as the shift in responsivity between October 302000 and July 22001 that is associated with a switch from A-side to B-side to A-side electronics [10], but other discontinuities are fitting artifacts within the uncertainty of the $\mathrm{OBC}$ measurements, and the derived trends may not adequately represent instrument response changes.

Another potential source of uncertainty is the degradation of the solar diffuser plate itself. At visible wavelengths, the reflectivity of the SD decreases with accumulated exposure to solar radiation, and that degradation has accelerated since May 2003 when an anomaly related to the SD door mechanism on MODIS-Terra led to the decision to leave the SD continuously exposed [11]. At $412 \mathrm{~nm}$, for example, the degradation rate of the diffuser plate reflectance has increased from 3\%/year to $10 \% / y e a r$, with total degradation as of early 2007 in excess of $30 \%$ [11]. This SD degradation is tracked and corrected via the solar diffuser stability monitor, but that correction also has uncertainties, and the reduction in available signal for calibration means that SD calibration uncertainty is increasing with time.

It is also evident from Fig. 1 that the amount of change in mirror-side reflectivity, as well as the relative difference between mirror sides (MS1 and MS2), is inconsistent between the SD and the SV measurement trends. The MODIS instrument views each on-orbit calibration source at a specific AOI on the scan mirror (Table 2), so changes in the relative difference between the SD and SV trends are an indication of temporal variability in RVS. MCST uses this information to track and correct for such variations [10], but that correction is limited to a simple rotation of the prelaunch RVS with time. Since the OBC can only track changes at two discrete AOIs, the onorbit adjustment can not account for changes in the shape of the RVS (MCST effectively rotates the prelaunch RVS shape around the SD AOI to match the relative change measured with respect to the SV AOI). Furthermore, given the overheating event, the prelaunch characterization of the RVS may not represent the at-launch RVS. The on-orbit calibration is also limited by the fact that the SV measurements saturate the detectors in the red and NIR ocean bands (longward of 650-nm, Tables 3 and 4), so no correction for temporal changes in red or NIR-band RVS are possible with this technique. Considering these limitations, some residual cross-scan calibration error is to be expected. 
Table 3. Change in MODIS-Terra System Response as of January 2007, relative to first on-orbit calibration. Measurements are derived from solar diffuser (SD) and lunar space-view (SV) trends, as defined in MCST calibration 5.0.6.27OC. Total \% change is shown for mirror-side 1 (MS1), followed by relative differences between mirror-side 2 (MS2) and MS1. ${ }^{a}$ Detectors saturate in lunar view.

\begin{tabular}{ccccc}
\hline Wavelength & SD MS1 & SD MS1-MS2 & SV MS1 & SV MS1-MS2 \\
\hline 412 & 36.3 & 5.0 & 40.6 & -5.0 \\
443 & 20.1 & 6.6 & 34.3 & 1.5 \\
488 & 10.7 & 2.7 & 20.0 & 3.8 \\
531 & 8.5 & 1.0 & 12.1 & 2.1 \\
551 & 14.6 & 0.6 & 16.8 & 1.4 \\
667 & -4.3 & 0.0 & $\mathrm{a}$ & $\mathrm{a}$ \\
678 & 6.9 & 0.0 & $\mathrm{a}$ & $\mathrm{a}$ \\
748 & 2.0 & 0.0 & $\mathrm{a}$ & $\mathrm{a}$ \\
869 & -1.0 & 0.1 & $\mathrm{a}$ & $\mathrm{a}$ \\
\hline
\end{tabular}

Table 4. Change in MODIS-Aqua System Response as of January 2007, relative to first on-orbit calibration. Measurements are derived from solar diffuser (SD) and lunar space-view (SV) trends, as defined in MCST calibration 5.0.7.22. Total \% change is shown for mirror-side 1 (MS1), followed by relative differences between mirror-side 2 (MS2) and MS1. ${ }^{a}$ Detectors saturate in lunar view.

\begin{tabular}{ccccc}
\hline Wavelength & SD MS1 & SD MS1-MS2 & SV MS1 & SV MS1-MS2 \\
\hline 412 & 13.1 & 0.6 & 19.7 & 1.2 \\
443 & 8.8 & 0.3 & 11.6 & 0.7 \\
488 & 5.4 & 0.1 & 5.8 & 0.3 \\
531 & 2.2 & 0.0 & 1.9 & 0.1 \\
551 & 1.8 & 0.0 & 1.4 & 0.0 \\
667 & -2.3 & -0.1 & $\mathrm{a}$ & $\mathrm{a}$ \\
678 & 0.9 & -0.1 & $\mathrm{a}$ & $\mathrm{a}$ \\
748 & 1.2 & -0.1 & $\mathrm{a}$ & $\mathrm{a}$ \\
869 & 0.4 & 0.0 & $\mathrm{a}$ & $\mathrm{a}$ \\
\hline
\end{tabular}

Finally, given the fact that the scan-mirror reflectivity has changed substantially over the Terra mission lifespan, presumably due to degradation of the mirror coatings, it is likely that the prelaunch characterization for polarization sensitivity as a function of mirror AOI is compromised. There is no on-board capability of MODIS to track changes in polarization sensitivity, and inaccuracy in polarization sensitivity knowledge can give rise to significant seasonal and geographically-dependent errors in the retrieved $L_{w}(\lambda)$ [9].

\section{IMPACT TO OCEAN COLOR RETRIEVALS}

Given the concerns and limitations with the MODIS-Terra prelaunch and on-orbit characterization, and NASA's goal of producing a long-term climate-research quality ocean color data record spanning multiple missions [12], a number of techniques have been applied to look for the signature of instrument calibration error in the derived ocean color trends. 


\subsection{Anomalous Temporal Trends}

Fig. 2 presents a comparison of MODIS-Terra and MODIS-Aqua $C_{a}$ and normalized waterleaving radiances, $L_{w n}(\lambda)$, as a function of time (where the $L_{w n}$ normalization adjusts $L_{w}$ to the conditions of a non-attenuating atmosphere with the Sun directly overhead [13]). This data was consistently processed for both sensors as described in Franz et al. (2005) [6], and vicariously calibrated using the approach detailed in Franz et al. (2007) [3], wherein a fixed vicarious gain is applied to the observed radiance of each sensor band. It is critical to understand that no time-dependent corrections have been applied for either sensor, other than those derived by MCST from the OBC measurements. Both sensors have had the benefit of equal treatment in processing and calibration. The trends in Fig. 2 were derived from globally distributed retrievals that were spatially averaged into approximately $9-\mathrm{km} \times 9-\mathrm{km}$ equal-area bins and then temporally averaged into consecutive 4-day composites at 32-day intervals. Specific bins were selected from each 4-day composite and spatially averaged to make the time-series, where the bin selection was limited to geographic locations with water depth greater than 1000 meters (deep water), and only those bins with valid data for both sensors (common bins) were included in the averages. The panels on the left show comparisons in absolute units, with the MODIS-Terra to MODIS-Aqua ratios shown on the right.
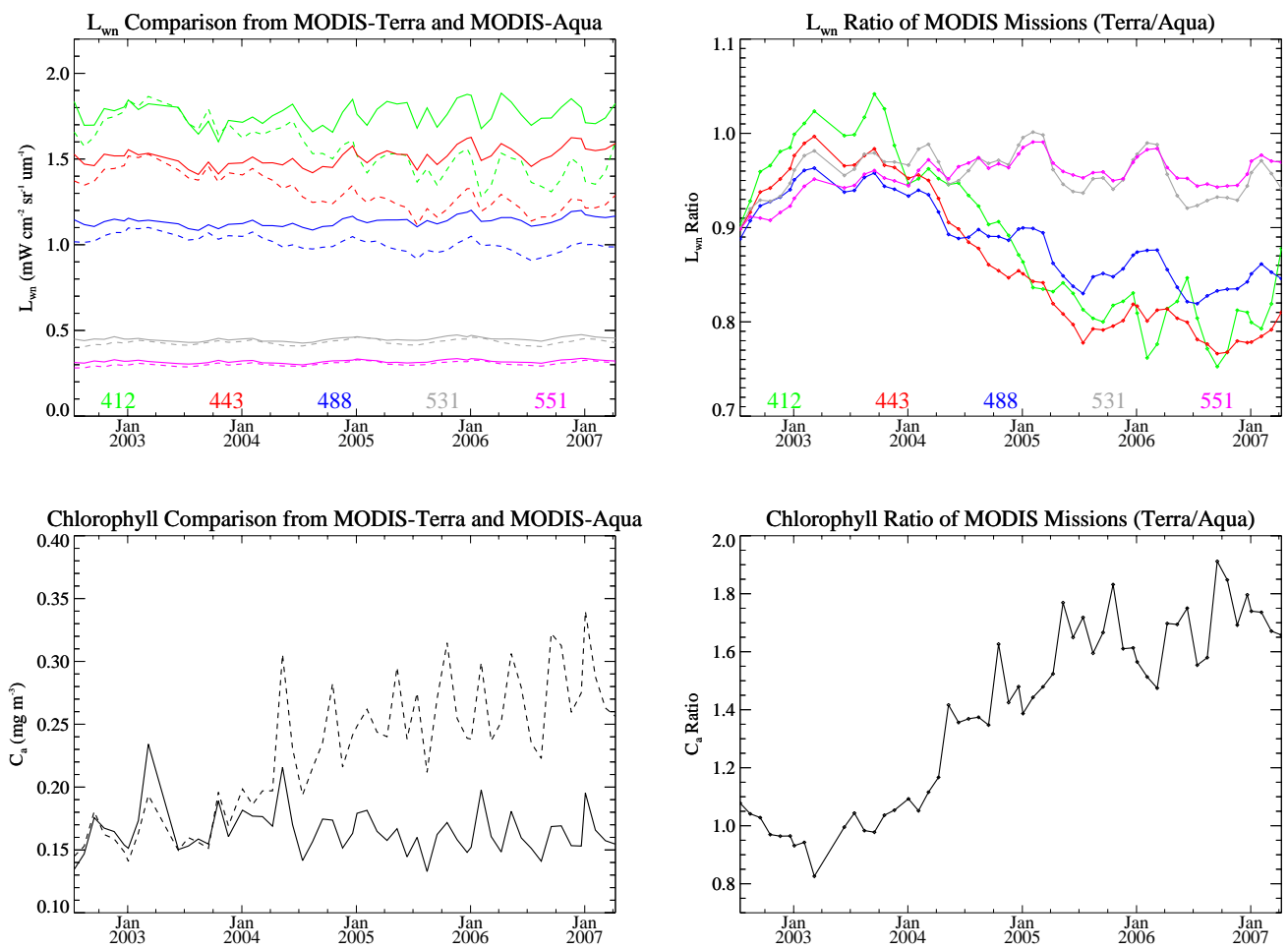

Fig. 2. Comparison of ocean color products derived from MODIS-Terra and MODISAqua. The upper-left panel shows $L_{w n}(\lambda)$ comparisons as a function of time for MODIS-Aqua (solid lines) and MODIS-Terra (dashed lines), with ratio of MODISTerra to MODIS-Aqua retrievals in the upper-right. The lower panels show the same for $C_{a}$. Trends are derived by averaging over deep-water retrievals in common bins. 
The trends in Fig. 2 indicate a strong departure in common ocean color retrievals between the two sensors. The deviations are much larger in the blue bands $(412-488 \mathrm{~nm})$ than in the green bands $(531-551 \mathrm{~nm})$. The relative trends in the $L_{w n}(\lambda)$ ratios vary from $10 \%$ low for all wavelengths at the beginning of the Aqua mission to approximately 5\% low in the green bands and 20\% low in the blue bands in January 2007, and the comparison trends indicate that the Aqua retrievals are relatively flat with time while the Terra retrievals are decreasing. If we assume the Aqua trends are more representative of truth, and consider Table 1, these results suggest a residual error in the temporal response for Terra of order $+0.4 \%$ (increasing) for the green bands and $-1.4 \%$ (decreasing) for the blue bands, relative to the calibration in July 2002 . This temporal variability might be due to errors or limitations of the OBC analysis, change in the polarization sensitivities relative to the prelaunch characterization, or more likely a combination of effects.

The lower panels of Fig. 2 show the comparison and ratio trends for $C_{a}$. In deep water, where mean $C_{a}$ is approximately $0.2 \mathrm{mg} \mathrm{m}^{-3}$, the $C_{a}$ algorithm used for operational MODIS processing (OC3) [4] is based on the ratio $L_{w n}(443) / L_{w n}(551)$, so the spectral dichotomy in the deviations of $L_{w n}(\lambda)$ gives rise to very large differences in the $C_{a}$ trends. The divergence in $C_{a}$ retrievals between Terra and Aqua is of order 60-70\%, which clearly must be corrected before the two sensors can be utilized in combination to assess long-term geophysical changes in world oceans. The $C_{a}$ trends also indicate an acceleration in the divergence between the two sensors beginning near the middle of 2003, which happens to correspond with the date of the aforementioned SD door anomaly on MODIS-Terra.

The above comparisons are limited to the time-span of the Aqua mission (May 2002 to present), and interpretations are based on the assumption that Aqua ocean color retrievals represent truth. To extend the evaluation, an alternative analysis was performed in which the temporal trends in the retrievals are evaluated relative to the mean seasonal cycle of those same retrievals. This is referred to as the seasonal anomaly. The seasonal cycle, which represents the dominant geophysical variability in the deep-water ocean color trends, was derived by averaging the data from common days over multiple years and fitting a smoothed cubic spline through the result. The plots on the left side of Fig. 3 show the $(9-\mathrm{km} \times 9-\mathrm{km})$ deep-water composite trends of $L_{w n}(412), L_{w n}(551)$, and $C_{a}$ after subtraction of the derived seasonal cycles. For comparison, the same analysis was applied to SeaWiFS and presented on the right side of Fig. 3, where the SeaWiFS trends are limited to the Terra mission time-span. The solid lines in each panel show the smoothed trend in seasonal anomalies (symbols), and the gray shaded areas indicate the range of linear trends that could be drawn through the anomalies (considering the $2 \sigma$ uncertainties in the linear fit coefficients). The SeaWiFS trends suggest a slightly increasing long-term trend in $L_{w n}(412)$, but the trends in all parameters are effectively consistent with the zero-slope case. In contrast, the Terra trends show significant temporal variability, thus corroborating the previous discussion regarding Fig. 2. The trend in MODIS-Terra $L_{w n}(551)$ is of particular note, as this band is only weakly dependent on changes in $C_{a}$ and hence there is no geophysical expectation of a substantial change in $L_{w n}(551)$ in deep-water (open ocean) conditions where $C_{a}$ is the primary water constituent modulating $L_{w n}(\lambda)$ (i.e., even if global bio-mass is changing, $L_{w n}(551)$ should be relatively constant, as is the case for SeaWiFS $\left.L_{w n}(555)\right)$ [14]. On a positive note, the Terra anomaly trends do not show any obvious discontinuities in late 2000 and mid 2001, suggesting that the MCST corrections for responsivity changes between A-side and B-side electronics were effective.

\subsection{Anomalous Geometry Trends and Mirror-Side Differences}

To investigate residual RVS error, the OBPG developed an analysis based on the idea that a multi-day temporal composite will tend to average-out the effects of scan geometry, as each bin location will be viewed at several different scan angles. Therefore, if we restrict the composite to bin locations with typically low spatial and temporal variability (i.e., deep-water with modest 

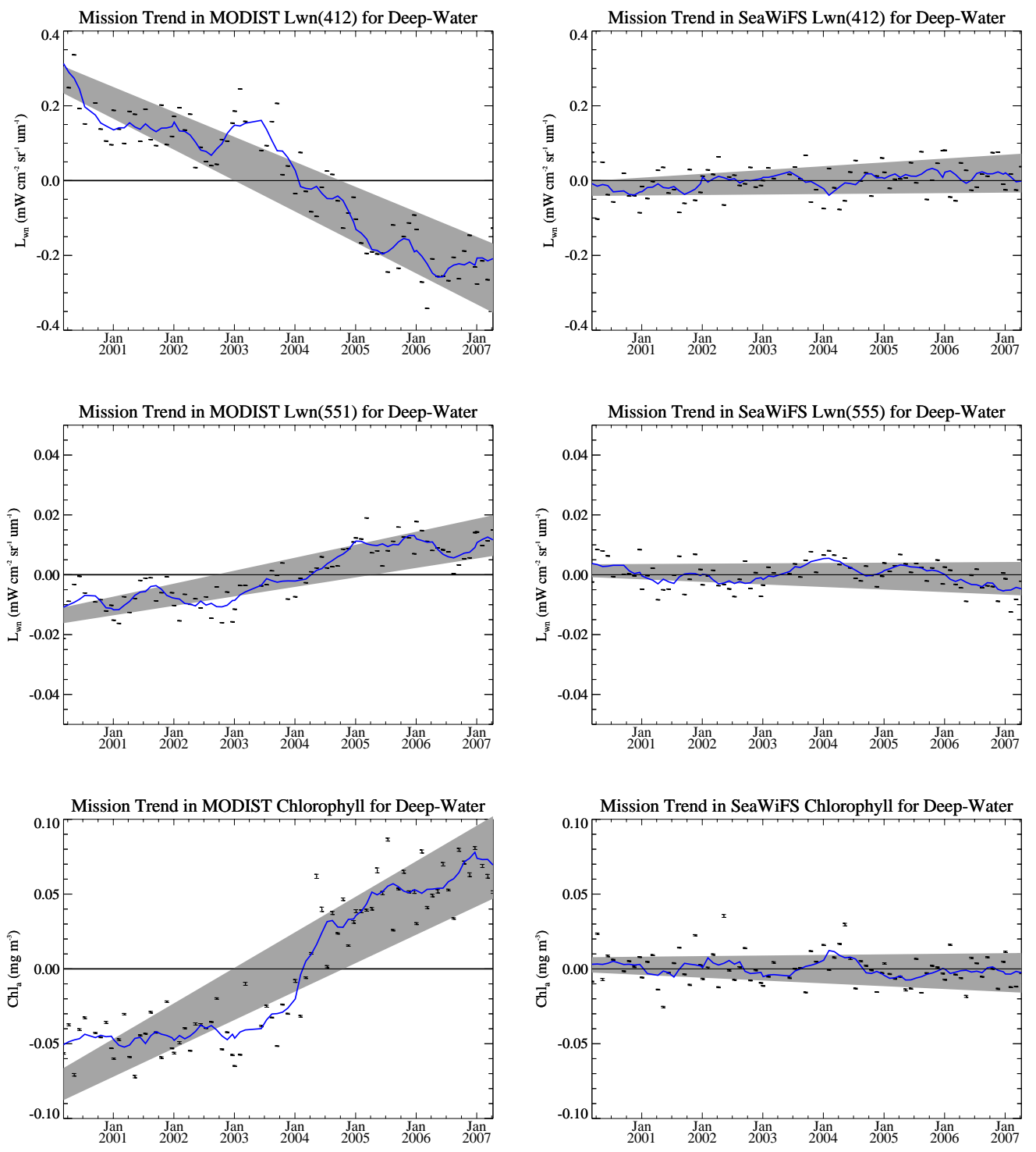

Fig. 3. Temporal trends in seasonal anomalies for ocean color products derived from MODIS-Terra and SeaWiFS. The symbols show mean deep-water values from consecutive 4-day composites, with error bars to indicate standard error on the mean, after subtraction of the deep-water seasonal cycle. The gray shaded area shows the range of linear fits that can be drawn through the data when considering the $2 \sigma$ error on the fit coefficients, and a 7-point filtered trend line is also provided to aid in visualization. Results are shown for MODIS-Terra and SeaWiFS $L_{w n}$ retreivals at 412 and $551 \mathrm{~nm}$ (555 $\mathrm{nm}$ for SeaWiFS), as well as for $C_{a}$. 
aerosol loads), we can use the temporal composite as relative truth for the assessment of residual RVS effects in the derived ocean color products. We start with global 7-day composites of MODIS $L_{w n}(\lambda)$ in equal area bins at 9-km x 9-km resolution, and match that to individual pixel retrievals taken from the central day of the composite period, where the pixels are at the native resolution of the MODIS ocean color bands (1-km x 1-km at nadir). We then sort the data by scan pixel number and mirror side and average the pixel-to-bin match-up ratios to derive the plots shown in Fig. 4. The symbols show the mean residual RVS trends for MS1 and MS2, with the associated standard deviation on the distributions presented as dashed lines. The upper two panels provide results for 23 September 2005, and the lower panels show the results exactly one year later. We stress that MODIS $L_{w n}$ retrievals are fully normalized to remove all known effects of solar and viewing path geometry, including subsurface bidirectional effects [13], so no residual scan-dependence is expected in these ratio plots. For the worst case of the 412 $\mathrm{nm}$ band, Fig. 4 shows residual peak-to-peak scan-dependent artifacts in $L_{w n}(412)$ of order $20 \%$ in 2005 , worsening to nearly $40 \%$ by 2006 . The error decreases rapidly with increasing wavelength, such that the residual RVS effects in $L_{w n}(551)$ approach zero. Compare these results to Fig. 5 that shows the identical analysis for MODIS-Aqua for the same day in 2006, using the same processing and calibration methods, with little or no residual RVS trend evident in either band.

Fig. 4 also demonstrates that mirror-side differences for Terra have not been fully corrected, and in fact the differences vary with scan pixel. The MCST correction for mirror-side reflectance, which is based primarily on observations at the SD and SV AOIs (scan pixels 23 and 979), can not adjust for nonlinear AOI-dependent trends. Furthermore, the relative differences we see between the mirror sides may be related to change in the polarization sensitivity, which can not be detected with the OBC. The 412-nm band is the most sensitive MODIS band to polarization [9], and we currently rely on the prelaunch characterization, in combination with the modeled atmospheric polarization, to derive the polarization correction.

\subsection{Residual Detector Striping}

Each ocean band on MODIS consists of 10 physical detectors oriented along the satellite track, allowing the instrument to collect $10 \times 1354$ observations of $L_{t}(\lambda)$ for each scan rotation. The residual RVS variabilities presented in Fig. 4 show a large spread in the distribution within each pixel, as indicated by the standard deviation lines. This is due in part to relative differences between the detectors, as demonstrated in Fig. 6 where the $L_{w n}$ retrievals have been further stratified to show the trends for the individual detectors within each band. MCST adjusts for differences in detector response through analysis of relative differences in the SD trends, and that correction has already been applied here. As with mirror-side differences, however, the SD-based correction can not account for detector differences that vary with scan-angle (mirror AOI). Such scan-dependent variability is evident in Fig. 6 for the detectors of the 412-nm band when viewing through MS2. Furthermore, each individual detector views the mirror at a slightly different AOI [15], so the inconsistency between detectors may also be attributable to error in the MS2 RVS or polarization knowledge. If left uncorrected, these detector-dependent errors give rise to cross-track striping in the ocean color imagery, and they complicate efforts to understand and correct for other systematic variabilities in instrument response. A similar analysis for MODIS-Aqua (not shown) reveals only a weak dependence in detector-to-detector relative calibration with scan angle, and thus the SD-based corrections are much more effective at removing detector striping artifacts for the sensor on Aqua. 

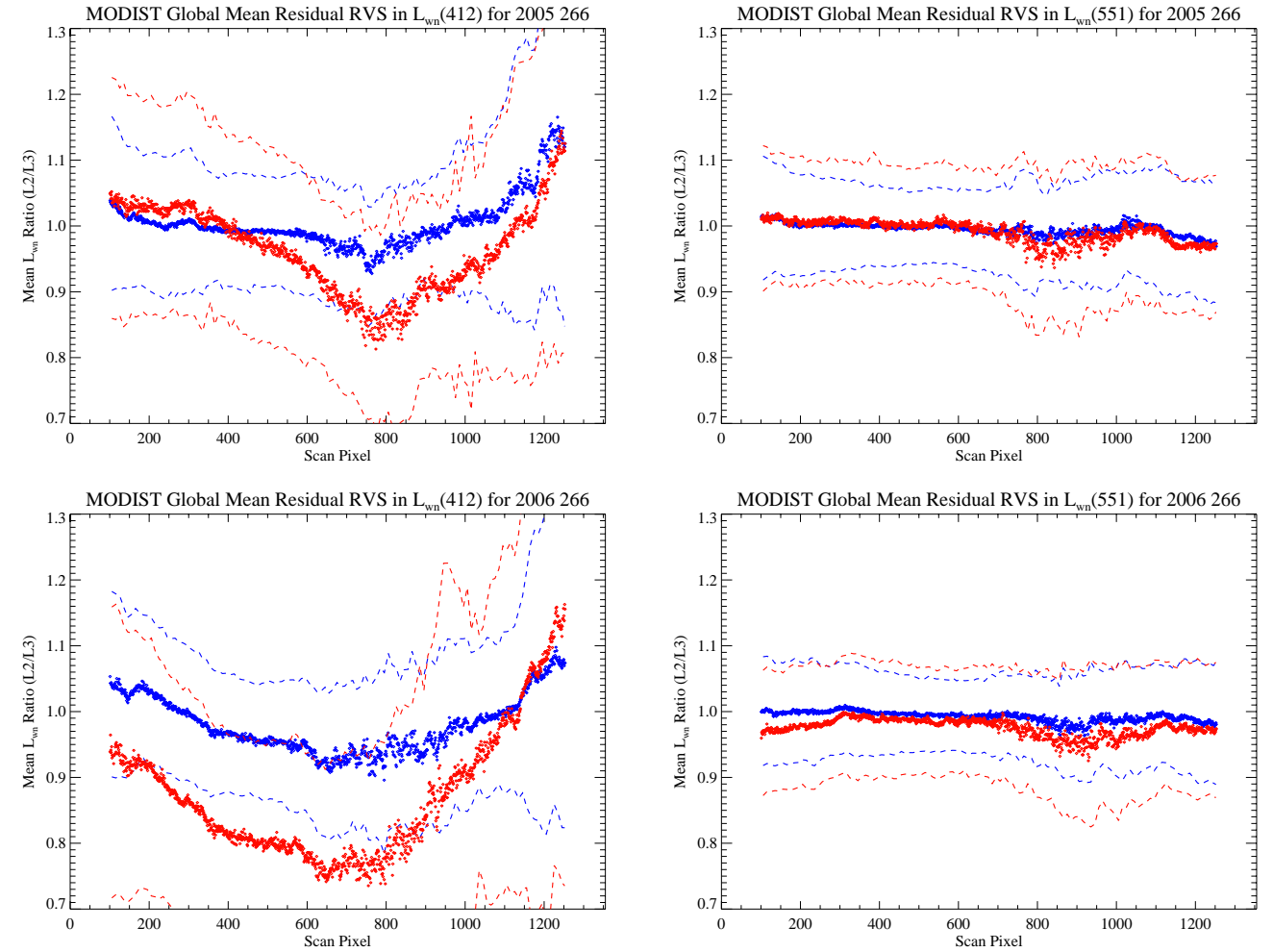

Fig. 4. Residual RVS trends in MODIS-Terra ocean color retrievals. Symbols provide mean geographic match-up ratios between individual scan pixel retrievals (L2) and the bounding 7-day global composite (L3), for all deep-water, clear atmosphere observations collected over a full day at a specific scan pixel and mirror-side. MS1 is in blue and MS2 is in red. Dashed lines indicate the standard deviation on the ratio distributions. Trends are shown for $L_{w n}$ at 412 and $551 \mathrm{~nm}$ as derived from data collected on 23 September (day 266) of 2005 and 2006. 

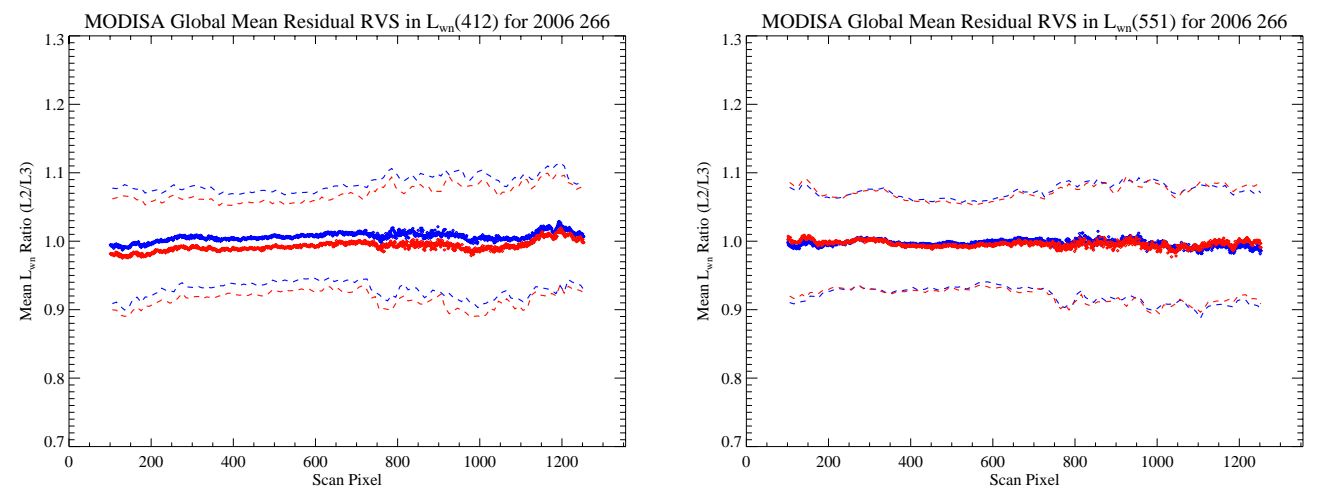

Fig. 5. Residual RVS trends in MODIS-Aqua ocean color retrievals. Trends as described in Fig. 4, but for MODIS-Aqua on 23 September of 2006 only.
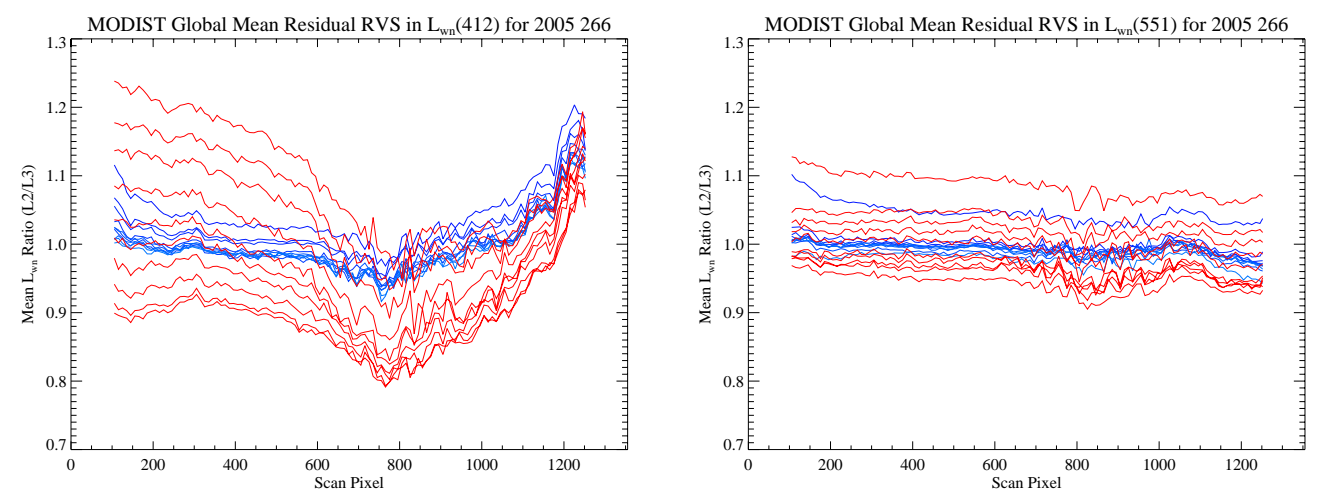

Fig. 6. Residual RVS trends in MODIS-Terra ocean color retrievals. Results as described for the upper two panels of Fig. 4, but separate trends are now shown for each of the 10 detectors within each ocean band (standard deviation lines dropped for clarity). Retrievals associated with MS1 are in blue, with MS2 results in red. These detector differences produce striping in the ocean color imagery.

\section{RECTIFICATION EFFORTS}

\subsection{Vicarious Calibration}

For all ocean color processing within the OBPG, a single-value (mission-averaged) vicarious calibration is employed to remove first-order bias between the $L_{w n}(\lambda)$ retrievals and ground truth measurements [3]. In principle, a consistently applied vicarious calibration should also serve to remove mean differences in the retrievals between sensors. In all ocean color results presented for MODIS-Terra, a vicarious calibration has been applied using the same techniques and calibration sites employed for MODIS-Aqua and SeaWiFS. This single-valued vicarious calibration, however, relies on the expectation that variations in instrument response with time, viewing geometry, mirror-side, and detector have already been corrected by the instrument prelaunch and on-orbit calibration. At present, such uncorrected variabilities add substantial noise to the vicarious calibration process, making consistent and reliable results for MODIS- 
Terra difficult to achieve. The vicarious calibration will be repeated as the instrument calibration and characterization is refined.

\subsection{Revised near-IR RVS Calibration}

In the OBPG atmospheric correction process, the NIR bands are utilized to determine aerosol type and concentration, and models are then used to extrapolate the retrieved aerosol radiance component of $L_{a t m}$ from the NIR to the visible bands [2]. Therefore, any error in the NIR calibration will contribute to error in $L_{w n}$ at all wavelengths. As previously noted, one limitation of the standard on-orbit calibration is the lack of information regarding the change in RVS at NIR wavelengths, as the SV observations of the Moon saturate the NIR detectors.

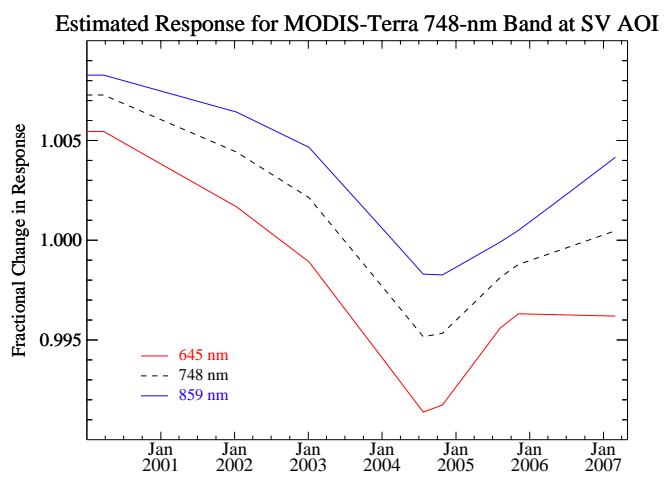

Fig. 7. Estimated change in the response of MODIS-Terra 748-nm band at the space view AOI. Solid lines show responsivity trends for the 645 and $859-\mathrm{nm}$ land bands, as derived from lunar measurements through the space view (SV) port. The dashed line indicates the change at $748 \mathrm{~nm}$, which is estimated by spectrally-weighted interpolation of the land bands because the 748-nm detectors saturate when viewing the Moon.

MCST and the OBPG are pursuing several strategies to address this limitation. One such approach takes advantage of additional MODIS bands at similar wavelengths that were designed with higher saturation radiances to support land and atmosphere applications. These include MODIS bands $1(645 \mathrm{~nm}), 2(859 \mathrm{~nm})$, and $17(904 \mathrm{~nm})$ that bound the saturating ocean bands at $667,678,748$, and $869 \mathrm{~nm}$. As an example, Fig. 7 shows the SV trends from the 645 and $859-\mathrm{nm}$ bands as a function of mission time, normalized to the prelaunch measurements at the SV AOI. Given that the RVS is an optical effect of the mirror (independent of the degradation of the detectors within each band), and that the prelaunch measurement for the 748-nm ocean color band falls between the bounding land bands, it is reasonable to assume that the change in RVS follows a simple wavelength dependence to first order. Thus, an estimated SV trend can be derived for the 748-nm ocean band using a weighted average of the temporal change measured in the 645 and $859-\mathrm{nm}$ land bands (with weighting determined by the relative proximity of the wavelengths). The estimated SV trend at $748 \mathrm{~nm}$, presented as the dashed line in Fig. 7, shows a maximum degradation of $1.2 \%$ relative to the prelaunch measurement at the SV AOI.

A revised MODIS-Terra calibration was developed to incorporate the estimated temporal dependence in the RVS of the red and NIR ocean bands $(667-869 \mathrm{~nm})$, which was previously assumed to be constant. The left panel of Fig. 8 shows the relative change in global mean deepwater $L_{w n}(\lambda)$ due to the incorporation of the time-dependent NIR RVS, expressed as the ratio 

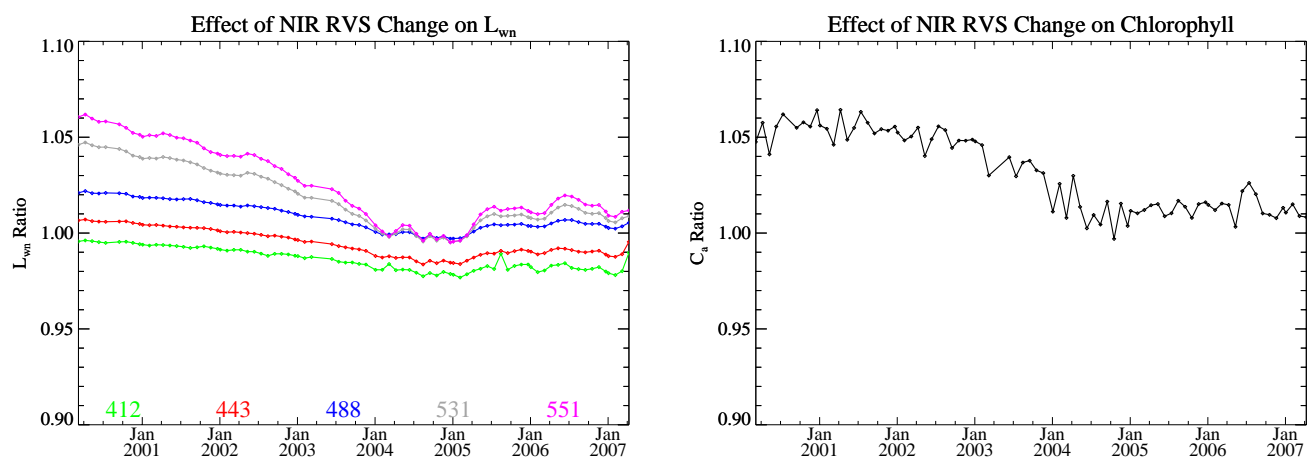

Fig. 8. Comparison of MODIS-Terra ocean color retrievals before and after correcting for temporal change in the NIR RVS. The left panel shows the impact to $L_{w n}(\lambda)$ while the right panel shows effect on $C_{a}$. Trends were derived by averaging over deep-water retrievals from consecutive 4-day global composites.

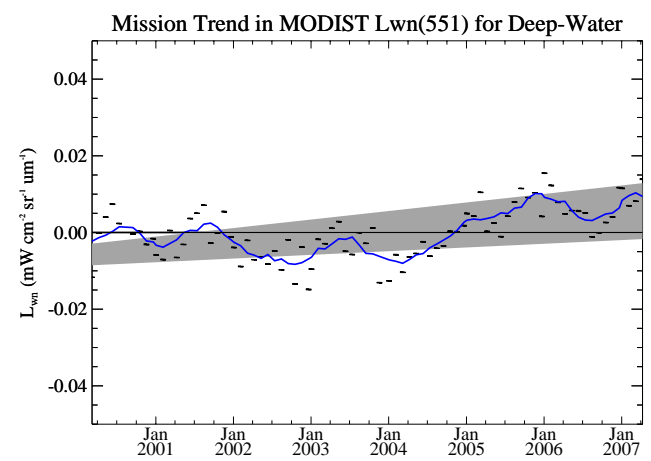

Fig. 9. Temporal trend in the seasonal anomaly for MODIS-Terra $L_{w n}(551)$ after correcting for temporal change in the NIR RVS. See Fig. 3 for description and comparison. The flatter trend is more consistent with geophysical expectation.

of $L_{w n}(\lambda)$ before the change to $L_{w n}(\lambda)$ after the change as a function of time. A similar plot for $C_{a}$ is provided in the right panel of Fig. 8. This analysis serves to illustrate the sensitivity of ocean color retrievals to changes in the NIR calibration. The impact to $L_{w n}(\lambda)$ and $C_{a}$ does alter the MODIS-Terra trends in the direction required to improve agreement with the SeaWiFS and MODIS-Aqua trends (Figs. 2 and 3), but the magnitude of the change is only a fraction of what is needed to mitigate the difference in blue to green $L_{w n}(\lambda)$ ratios that are driving the $C_{a}$ trend error in MODIS-Terra. It is worth noting, however, that the NIR RVS adjustment was sufficient to largely flatten the seasonal anomaly trend at $L_{w n}(551)$, making the long-term trend more consistent with geophysical expectation, as shown in Fig. 9.

\subsection{Vicarious RVS and Polarization Characterization}

As previously noted, another major limitation of the MODIS OBC is the inability to track variations in the polarization sensitivity of the mirror or changes in the shape of the RVS relative 
to the prelaunch characterization. To address this issue, the OBPG developed a vicarious technique for on-orbit characterization of the instrument response, wherein the observed radiance at the TOA is written as

$L_{t}(\lambda)=M_{11} L_{I}(\lambda)+M_{12}\left[L_{Q}(\lambda) \cos 2 \alpha-L_{U}(\lambda) \sin 2 \alpha\right]+M_{13}\left[L_{Q}(\lambda) \sin 2 \alpha+L_{U}(\lambda) \cos 2 \alpha\right]$,

with $M_{11}, M_{12}$, and $M_{13}$ representing the instrument Mueller matrix, $L_{I}(\lambda), L_{Q}(\lambda)$, and $L_{U}(\lambda)$ the components of the Stokes vector for linearly polarized radiance exiting the TOA, and $\alpha$ a frame rotation angle [8]. In the context of the previous discussion, $M_{11}$ encompasses the instrument RVS and $M_{12}$ and $M_{13}$ represent the polarization sensitivity. These are the three quantities we wish to determine for each sensor wavelength, detector number, mirror-side, and scan pixel. To achieve this, we need to know $L_{I}(\lambda), L_{Q}(\lambda)$, and $L_{U}(\lambda)$ over a wide range of viewing geometries and atmospheric polarization states, and then optimize the Mueller matrix to minimize the difference between modeled and observed $L_{t}(\lambda)$ over the associated range in mirror AOI and polarization states.
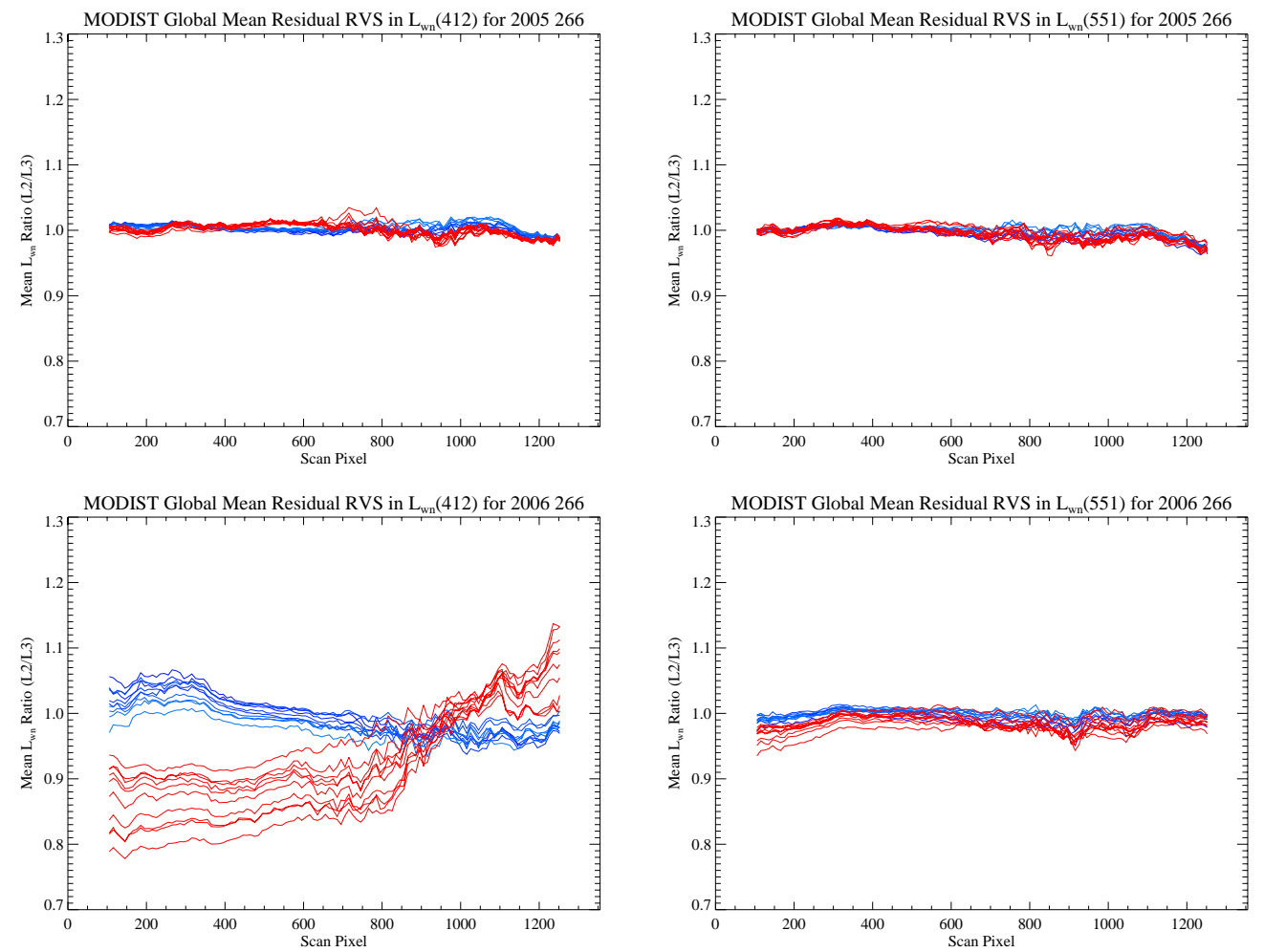

Fig. 10. Residual RVS trend per detector for MODIS-Terra ocean color retrievals after application of the vicariously-determined instrument characterization model. See Figs. 4 and 6 for plot description and comparison.

For a given set of viewing and solar radiant path geometries, the polarization components $L_{Q}(\lambda)$ and $L_{U}(\lambda)$ can be readily estimated from radiative transfer models [8]. To estimate $L_{I}(\lambda)$, the OBPG vicarious calibration process [3] is utilized in combination with a global 
distribution of SeaWiFS $L_{w n}(\lambda)$ retrievals. The vicarious calibration is an inversion of the atmospheric correction model wherein TOA radiance is predicted for a given water-leaving radiance. The advantage of using SeaWiFS is that the retrievals are globally distributed, unlike the available in situ data set [14], thus allowing for calibration match-ups for all detectors and both mirror-sides over a wide range of radiant path geometries. In addition, SeaWiFS should be free of any polarization artifacts, as the instrument was designed to minimize polarization sensitivity [16]. It should be emphasized that this approach is not intended as an absolute crosscalibration, as the final step in the process is to perform the single-value vicarious calibration to ground truth (Section 4.1).

The vicarious characterization process was performed for a global distribution of MODISTerra $L_{t}(\lambda)$ observations from 19 September 2005, using a bounding 7-day composite of SeaWiFS deep-water $L_{w n}(\lambda)$ retrievals as the truth field. The revised instrument characterization was then applied in the processing of MODIS-Terra data from 23 September of 2005 and 2006. The results, as presented in Fig. 10, show a dramatic improvement in residual RVS for the 2005 case, which is close to the date for which the revised characterization was derived (compare to Fig. 6). The improvement is not as complete for the 2006 case, which just indicates that the characterization must be performed on a periodic basis to track temporal change. More work is needed to produce and evaluate a time-series of Mueller matrices derived over the Terra mission life-span, but these preliminary results are promising.

\section{CONCLUSIONS AND FUTURE PLANS}

The OBPG is currently producing compatible ocean color products from the MODIS-Aqua and SeaWiFS sensors using common software and algorithms [6]. With the same processing approach, we demonstrated that ocean color retrievals derived from MODIS-Terra show significant temporal and geometric variabilities that are inconsistent with the other sensors, and we provided evidence to suggest that these variabilities are related to issues and limitations of the MODIS-Terra prelaunch and on-orbit calibration and characterization. Of particular concern is the spectrally-bifurcated temporal trends observed in the $L_{w n}(\lambda)$ retrievals that result in 60$70 \%$ errors in global mean $C_{a}$ trends. In addition, residual scan-dependent variabilities as large as $20-40 \%$ in $L_{w n}(\lambda)$ were revealed, as well as significant differences between mirror sides and between detectors. The largest errors were seen in the shortest ocean band wavelengths (412 $488 \mathrm{~nm}$ ), where polarization sensitivities are highest and degradations in system response and SD degradation are most severe.

We discussed several successful OBPG efforts to reduce instrumental errors in the ocean color retrievals, including a correction for temporal variability in the NIR RVS based on OBC measurements, and a vicarious technique for deriving the instrument RVS and polarization sensitivities on-orbit. The modified NIR RVS was found to reduce the long-term temporal trends for $L_{w n}(551)$ to within geophysical expectations, and the vicarious instrument characterization showed a dramatic improvement in residual RVS, detector striping, and mirror-side difference for our limited test case. We plan to develop and evaluate a full-mission vicarious instrument characterization for MODIS-Terra in the coming months. We will also continue working with MCST on the re-analysis and evaluation of the OBC measurements, with the goal of identifying alternative interpretations that may better represent temporal changes in system response. In the interim, we recommend that ocean color products generated from MODIS-Terra be restricted to qualitative applications.

It is also worth noting that some of the concerns with MODIS-Terra calibration and characterization are relevant to MODIS-Aqua. The Aqua sensor is degrading at a similarly rapid rate, and changes in polarization sensitivity, for example, are likely to impact ocean color retrievals in the future. Lessons learned from efforts to improve the performance of MODIS-Terra for ocean color may well be useful for application to MODIS-Aqua. 


\section{Acknowledgments}

The authors wish to thank the MODIS Calibration Support Team under the leadership of X. Xiong for continued support of MODIS instrument calibration and many useful discussions and ideas for interpreting and improving the results presented here. We also acknowledge the work of R. Evans and colleagues at the University of Miami, Rosenstiel School of Marine Sciences, through which a number of the issues with MODIS-Terra for ocean color were first recognized. Finally, we want to thank our colleagues at the OBPG for processing support and helpful discussions on the development and interpretation of this analysis, and particularly S. Bailey for generation and analysis of the vicarious calibrations.

\section{References}

[1] W. E. Esaias, M. R. Abbot, I. J. Barton, O. B. Brown, J. W. Campbell, K. L. Carder, D. K. Clark, R. H. Evans, F. E. Hoge, H. R. Gordon, W. M. Balch, R. Letelier, and P. J. Minnett, "An overview of MODIS capabilities for ocean science observations," IEEE Trans. Geosci. Rem. Sens. 36, 1250-1265 (1998) [doi:10.1109/36.701076].

[2] H. R. Gordon and M. Wang, "Retrieval of water-leaving radiance and aerosol optical thickness over the oceans with SeaWiFS: A preliminary algorithm," Appl. Opt. 33, 443-452 (1994).

[3] B. A. Franz, S. W. Bailey, P. J. Werdell, and C. R. McClain, "Sensor-independent Approach to the Vicarious Calibration of Satellite Ocean Color Radiometry," Appl. Opt. 46, 5068-5082 (2007) [doi:10.1364/AO.46.005068].

[4] J. E. O'Reilly, S. Maritorena, B. G. Mitchell, D. A. Siegel, K. L. Carder, S. A. Garver, M. Kahru, and C. R. McClain, "Ocean color chlorophyll algorithms for SeaWiFS," J. Geophys. Res 103, 24937-24953 (1998) [doi:10.1029/98JC02160].

[5] C. R. McClain, M. L. Cleave, G. C. Feldman, W. W. Gregg, S. B. Hooker, and N. Kuring, "Science quality SeaWiFS data for global biosphere research," Sea Tech. 39(9), 10-16 (1998).

[6] B. A. Franz, P. J. Werdell, G. Meister, S. W. Bailey, R. E. Eplee, G. C. Feldman, E. Kwiatkowska, C. R. McClain, F. S. Patt, and D. Thomas, "The continuity of ocean color measurements from SeaWiFS to MODIS," Proc. SPIE 5882(1), 58820W (2005) [doi:10.1117/12.620069].

[7] X. Xiong and W. L. Barnes, "An overview of MODIS radiometric calibration and characterization," Adv. Atmos. Sci. 23(1), 69-79 (2006) [doi:10.1007/s00376-006-0008-3].

[8] H. R. Gordon, T. Du, and T. Zhang, "Atmospheric correction of ocean color sensors: analysis of the effects of residual instrument polarization sensitivity," Appl. Opt. 36(27), 6938-6948 (1997).

[9] G. Meister, E. J. Kwiatkowska, B. A. Franz, F. S. Patt, G. C. Feldman, and C. R. McClain, "Moderate-resolution imaging spectroradiometer ocean color polarization correction," Appl. Opt. 44(26), 5524-5535 (2005) [doi:10.1364/AO.44.005524].

[10] J. Sun, X. Xiong, B. Guenther, and W. Barnes, "Radiometric stability monitoring of the MODIS reflective solar bands using the moon," Metrologia 40, S85-S88 (2003) [doi:10.1088/0026-1394/40/1/319].

[11] X. Xiong, H. Erives, S. Xiong, X. Xie, J. Esposito, J. Sun, and W. Barnes, "Performance of Terra MODIS solar diffuser and solar diffuser stability monitor," Proc. SPIE 5882(1), 58820S (2005) [doi:10.1117/12.615334].

[12] C. R. McClain, S. B. Hooker, G. C. Feldman, and P. Bontempi, "Satellite data for ocean biology, biogeochemistry, and climate research," EOS Trans. AGU 87(34), 337-343 (2006) [doi:10.1029/2006EO340002].

[13] H. R. Gordon, "Normalized water-leaving radiance: revisiting the influence of surface roughness," Appl. Opt. 44(2), 241-248 (2005) [doi:10.1364/AO.44.000241]. 
[14] P. Werdell and S. Bailey, "An improved in-situ bio-optical data set for ocean color algorithm development and satellite data product validation," Rem. Sens. Environ. 98, 122-140 (2005) [doi:10.1016/j.rse.2005.07.001].

[15] G. Meister, E. J. Kwiatkowska, and C. R. McClain, "Analysis of image striping due to polarization correction artifacts in remotely sensed ocean scenes," Proc. SPIE 6296(1), 629609 (2006) [doi:10.1117/12.678309].

[16] R. A. Barnes, A. W. Holmes, W. L. Barnes, W. E. Esaias, and T. Svitek, "SeaWiFS prelaunch radiometric calibration and spectral characterization," NASA Tech. Memo. 104566, National Aeronautics and Space Administration, Goddard Space Flight Center, Greenbelt, MD (1995). 